The Journal of $\mathbf{N}_{\text {onlinear }} \mathbf{S}$ ciences and Applications

http://www.tjnsa.com

\title{
FUZZY NONLINEAR OPTIMIZATION
}

\begin{abstract}
S.H. NASSERI *
ABstract. In this paper, we define a fuzzy version of the quadratic optimization problem and then give a method to solve these problems by using linear ranking functions. The method will be discussed in details.
\end{abstract}

\section{INTRODUCTION}

Fuzzy set theory has been applied to many disciplines such as control theory and management sciences, mathematical modeling, operations research and many industrial applications. The concept of fuzzy mathematical programming on general level was first proposed by Tanaka et al. [11] in the framework of the fuzzy decision of Bellman and Zadeh [2]. Afterwards, many authors considered various types of the fuzzy linear programming (FLP) problems and proposed several approaches for solving these problems [4], [7, 8, 9, 10]. Some authors used the concept of comparison of fuzzy numbers for solving fuzzy linear programming problems. In effect, most convenient methods are based on the concept of comparison of fuzzy numbers by use of ranking functions [4], [7, 8, 9, 10. Usually in such methods authors define a crisp model which is equivalent to the FLP problem and then use the optimal solution of the model as the optimal solution of the FLP problem. A review of some common methods for ranking fuzzy subsets of the unit interval can be seen in [3]. Moreover, a review of the literature concerning fuzzy mathematical programming as well as comparison of fuzzy numbers can be seen in Klir and Yuan [6] and also Lai and Hwang [7]. In this paper, we consider a fuzzy version of the quadratic programming problems and then present a method to solve these problems by using linear ranking functions as a convenient tools for ordering fuzzy numbers.

Date: Received: 7 July 2008; Revised: 26 December 2008.

* Corresponding author. nasseri@umz.ac.ir (Hadi Nasseri).

Key words and phrases. Fuzzy numbers, ranking function, quadratic programming. 
The paper is organized in 5 Sections. In Section 2, we give some necessary notations and definitions of fuzzy set theory and fuzzy arithmetic. Section 3 provides a discussion of fuzzy numbers and linear ranking functions for ordering them. In particular, Yager's linear ranking function for ordering trapezoidal fuzzy numbers is emphasized. We define a fuzzy quadratic programming (FQP) problems in Section 4, and focus on solving theses problems. Moreover, we give a key theorem to state some conditions of the existence of a unique solution. Finally, we conclude in Section 5.

\section{Definitions and Notations}

We review the fundamental notions of fuzzy set theory, initiated by Bellman and Zadeh [2].

Definition 2.1. Let $X$ be the universal set. $\tilde{A}$ is called a fuzzy set in $X$ if $\tilde{A}$ is a set of ordered pairs

$$
\tilde{A}=\left\{\left(x, \mu_{\tilde{A}}(x)\right) \mid x \in X\right\}
$$

where $\mu_{\tilde{A}}(x)$ is the membership function of $\tilde{A}$.

Definition 2.2. The $\lambda$-level set of $\tilde{A}$ is the set

$$
\tilde{A}_{\lambda}=\left\{x \in X \mid \mu_{\tilde{A}}(x) \geq \lambda\right\}
$$

where $\lambda \in(0,1]$. The lower and upper bounds of any $\lambda$-level set $\tilde{A}_{\lambda}$ are represented by finite numbers $\inf _{x \in \tilde{A}_{\lambda}}$ and $\sup _{x \in \tilde{A}_{\lambda}}$.

Definition 2.3. A convex fuzzy set $\tilde{A}$ on $\mathbb{R}$ is a fuzzy number if the following conditions hold:

(a) Its membership function is piecewise continuous.

(b) There exist three intervals $[a, b],[b, c]$ and $[c, d]$ such that $\mu_{\tilde{A}}$ is increasing on $[a, b]$, equal to 1 on $[b, c]$, decreasing on $[c, d]$ and equal to 0 elsewhere.

Definition 2.4. Let $\tilde{A}=\left(a^{L}, a^{U}, \alpha, \beta\right)$ denote the trapezoidal fuzzy number, where $\left(a^{L}-\alpha, a^{U}+\beta\right)$ is the support of $\tilde{A}$ and $\left[a^{L}, a^{U}\right]$ its core.

Remark 2.5. We denote the set of all trapezoidal fuzzy numbers by $F(\mathbb{R})$.

We next define arithmetic on trapezoidal fuzzy numbers. Let $\tilde{a}=\left(a^{L}, a^{U}, \alpha, \beta\right)$ and $\tilde{b}=\left(b^{L}, b^{U}, \gamma, \theta\right)$ be two trapezoidal fuzzy numbers. Define,

$$
\begin{aligned}
& x>0, x \in \mathbb{R} ; \quad x \tilde{a}=\left(x a^{L}, x a^{U}, x \alpha, x \beta\right) \\
& x<0, x \in \mathbb{R} ; \quad x \tilde{a}=\left(x a^{U}, x a^{L},-x \beta,-x \alpha\right) \\
& \tilde{a}+\tilde{b}=\left(a^{L}+b^{L}, a^{U}+b^{U}, \alpha+\gamma, \beta+\theta\right) \\
& \tilde{a}-\tilde{b}=\left(a^{L}-b^{U}, a^{U}-b^{L}, \alpha+\theta, \beta+\gamma\right)
\end{aligned}
$$

We point out that the arithmetic on trapezoidal fuzzy numbers follow the Extension Principle (see [7]). 


\section{RANKING FUnCTIONS}

An effective approach for ordering the elements of $F(\mathbb{R})$ is to define a ranking function $R: F(\mathbb{R}) \rightarrow \mathbb{R}$ which maps each fuzzy number into the real line, where a natural order exists.

We define orders on $F(\mathbb{R})$ by:

$$
\begin{array}{lll}
\tilde{a} \succeq \tilde{b} & \text { if and only if } & R(\tilde{a}) \geqslant R(\tilde{b}) \\
\tilde{a} \succ \tilde{b} & \text { if and only if } & R(\tilde{a})>R(\tilde{b}) \\
\tilde{a} \simeq \tilde{b} & \text { if and only if } & R(\tilde{a})=R(\tilde{b})
\end{array}
$$

where $\tilde{a}$ and $\tilde{b}$ are in $F(\mathbb{R})$. Also we write $\tilde{a} \preceq \tilde{b}$ if and only if $\tilde{b} \succeq \tilde{a}$.

We restrict our attention to linear ranking functions, that is, a ranking function $R$ such that

$$
R(k \tilde{a}+\tilde{b})=k R r(\tilde{a})+R(\tilde{b})
$$

for any $\tilde{a}$ and $\tilde{b}$ belonging to $F(\mathbb{R})$ and any $k \in \mathbb{R}$ (see in $[8]$ ).

Remark 3.1. For any trapezoidal fuzzy number $\tilde{a}$, the relation $\tilde{a} \succeq \tilde{0}$ holds, if there exist $\varepsilon \geqslant 0$ and $\alpha \geqslant 0$ such that $\tilde{a} \succeq(-\varepsilon, \varepsilon, \alpha, \alpha)$. We realize that $R(-\varepsilon, \varepsilon, \alpha, \alpha)=$ 0 (we also consider $\tilde{a} \simeq \tilde{0}$ if and only if $R(\tilde{a})=0$ ). Thus, without loss of generality, throughout the paper we let $\tilde{0}=(0,0,0,0)$ as the zero trapezoidal fuzzy number.

The following lemma is now immediately in hand.

Lemma 3.2. Let $R$ be any linear ranking function. Then,

(i) $\tilde{a} \succeq \tilde{b}$ if and only if $\tilde{a}-\tilde{b} \succeq \tilde{0}$ if and only if $-\tilde{b} \succeq-\tilde{a}$.

(ii) If $\tilde{a} \succeq \tilde{b}$ and $\tilde{c} \succeq \tilde{d}$, then $\tilde{a}+\tilde{c} \succeq \tilde{b}+\tilde{d}$.

We consider the linear ranking functions on $F(\mathbb{R})$ as:

$$
R(\tilde{a})=c_{L} a^{L}+c_{U} a^{U}+c_{\alpha} \alpha+c_{\beta} \beta,
$$

where $\tilde{a}=\left(a^{L}, a^{U}, \alpha, \beta\right)$, and $c_{L}, c_{U}, c_{\alpha}, c_{\beta}$ are constants, at least one of which is nonzero. A special version of the above linear ranking function was first proposed by Yager [12] (see also [5]) as follows:

$$
R(\tilde{a})=\frac{1}{2} \int_{0}^{1}\left(\inf \tilde{a}_{\lambda}+\sup \tilde{a}_{\lambda}\right) d \lambda
$$

which reduces to

$$
R(\tilde{a})=\frac{a^{L}+a^{U}}{2}+\frac{1}{4}(\beta-\alpha) .
$$

Then, for trapezoidal fuzzy numbers $\tilde{a}=\left(a^{L}, a^{U}, \alpha, \beta\right)$ and $\tilde{b}=\left(b^{L}, b^{U}, \gamma, \theta\right)$, we have

$$
\tilde{a} \succeq \tilde{b} \quad \text { if and only if } \quad a^{L}+a^{U}+\frac{1}{2}(\beta-\alpha) \geqslant b^{L}+b^{U}+\frac{1}{2}(\theta-\gamma) .
$$




\section{Fuzzy Quadratic Programming}

A fuzzy quadratic programming (FQP) problem is defined as:

$$
\begin{array}{ll}
\text { minimize } & \frac{1}{2} x^{T} Q x+x^{T} c \\
\text { subject to } & \tilde{A} x \simeq \tilde{b}
\end{array}
$$

where $\tilde{b} \in\left(F(\mathbb{R})^{m}, \tilde{A}=\left[\tilde{a}_{i j}\right] \in(F(\mathbb{R}))\right)^{m \times n}, c^{T} \in \mathbb{R}^{n}, Q \in \mathbb{R}^{m \times n}$ are given and $x \in\left(\mathbb{R}^{n}\right.$ is to be determined.

The following theorem, which is an extension of the concepts concerning to the fuzzy arithmetic and linear ranking functions on nonlinear programming with fuzzy numbers, will be useful for solving the FQP problems.

Theorem 4.1. The following quadratic programming problem and FQP problem are equivalent.

$$
\begin{aligned}
& \text { minimize } \frac{1}{2} x^{T} Q x+x^{T} c \\
& \text { subject to } A x=b,
\end{aligned}
$$

where $a_{i j}$ and $b_{i}, i=1, \ldots, m, j=1, \ldots, n$ are real numbers corresponding to the fuzzy numbers $\tilde{a}_{i j}$ and $\tilde{b}_{i}, i=1, \ldots, m, j=1, \ldots, n$ with respect to a given linear ranking function, respectively.

Proof. Let $Q_{1}$ and $Q_{2}$ be sets of all feasible solutions of (4.1) and (4.2), respectively. So it is enough to show that $Q_{1}=Q_{2}$. Now we know that $x \in Q_{1}$ if and only if $\tilde{A} x \simeq \tilde{b}$, if and only if

$$
R\left(\sum_{j=1}^{n} \tilde{a}_{i j} x_{j}\right)=R\left(\tilde{b}_{i}\right), i=1, \ldots, m,
$$

if and only if

if and only if

$$
\sum_{j=1}^{n} R\left(\tilde{a}_{i j} x_{j}\right)=R\left(\tilde{b}_{i}\right), i=1, \ldots, m
$$

or

$$
\sum_{j=1}^{n} a_{i j} x_{j}=b_{i}, i=1, \ldots, m,
$$

$$
A x=b,
$$

where $a_{i j}=R\left(\tilde{a}_{i j}\right), b_{i}=\operatorname{Rr}\left(\tilde{b}_{i}\right)$, for all $j=1, \ldots, n$, and $i=1, \ldots, m$, if and only if $x \in Q_{2}$. Hence $Q_{1}=Q_{2}$. And this shows that the two mentioned quadratic problems are equivalent. The proof complete now.

The mentioned theorem represent that for solving an FQP problem it is enough to solve an equivalent quadratic programming in crisp environment. So here we deal with on these problems.

It is simple to show the FQP problem has a unique solution if the matrix $A$ is of full rank and the matrix $Q$ is positive definite on the subspace $M=\{x \mid A x=0\}$. 
Now consider the Lagrange necessary conditions for this problem as follows:

$$
\begin{aligned}
Q x+A^{T} \lambda+c & =0 \\
A x-b & =0 .
\end{aligned}
$$

These correspond to the general condition Lagrange (see in [1]), and in this case they comprise an $(n+m)$-dimensional linear system of equations. A natural question is whether the system is nonsingular. The following Theorem shows that the system is indeed nonsingular under the conditions stated above.

Theorem 4.2. Let $Q$ and $A$ be $n \times n$ and $m \times n$ matrices, respectively. Suppose that $A$ has rank $m$ and that $Q$ is positive definite on the subspace $M=\{x \mid A x=$ $0\}$. Then the matrix

$$
\left(\begin{array}{cc}
Q & A^{T} \\
A & 0
\end{array}\right)
$$

is nonsingular.

Proof. Suppose $(x, y) \in \mathbb{R}^{n+m}$ is such that

$$
\begin{aligned}
Q x+A^{T} y & =0 \\
A x & =0 .
\end{aligned}
$$

Multiplication of the first equation by $x^{T}$ yields

$$
x^{T} Q x+x^{T} A^{T} y=0,
$$

and substitution of $A x=0$ yields $x^{T} Q x=0$. However, clearly $x \in M$, and thus the hypothesis on $Q$ together with $x^{T} Q x=0$ implies that $x=0$. It then follows that the first equation that $A^{T} y=0$. The full rank condition on $A$ then implies that $y=0$. Thus the only solution to (4.4) is $x=0, y=0$.

Under the assumptions of Theorem 4.2, there are several methods for solving the system (4.3). As a general rules it is most efficient to use factorization methods (such as $L U$ decomposition) that exploit the structure of the solving linear equations can be used.

Remark 4.3. Note that if, as is often the case, the matrix $Q$ is actually positive definite (over the whole space), then an explicit formula for the solution of the system can be easily derived as follows:

From the first equation in (4.3) we have

$$
x=-Q^{-1} A^{T} \lambda-Q^{-1} c .
$$

Substitution of this into the second equation then yields

$$
-A Q^{-1} A^{T} \lambda-A Q^{-1} c-b=0,
$$

from which we immediately obtain

$$
\lambda=-\left(A Q^{-1} A^{T}\right)^{-1}\left[A Q^{-1} c+b\right]
$$

and

$$
\begin{aligned}
x & =Q^{-1} A^{T}\left(A Q^{-1} A^{T}\right)^{-1}\left[A Q^{-1} c+b\right]-Q^{-1} c \\
& =-Q^{-1}\left[I-A^{T}\left(A Q^{-1} A^{T}\right)^{-1} A Q^{-1}\right] c+Q^{-1} A^{T}\left(A Q^{-1} A^{T}\right)^{-1} b .
\end{aligned}
$$


This representation is useful in theoretical developments, although in practice the solution may be calculated by some other method as discussed above.

\section{Conclusions}

Using the linear ranking functions is a convenient method for solving fuzzy linear programming problems. In this paper, we defined the fuzzy quadratic programming problems and discussed how can solve FQP problems by using linear ranking functions.

Acknowledgements: The Author thanks to the Research Center of Algebraic Hyperstructures and Fuzzy Mathematics for its partly supports.

\section{REFERENCES}

1. M. Bazaraa, H. Sherali and C.M. Shetty, Nonlinear Programming: Theory and Algorithms, Third Edition, John Wiley, 2006. 4

2. R.E. Bellman and L.A. Zadeh, Decision making in a fuzzy environment, Management Sci. 17 (1970) 141-164. 1, 2

3. G. Bortolan and R. Degani, A review of some methods for ranking fuzzy numbers, Fuzzy Sets and Systems 15 (1985) 1-19. 1

4. L. Campos and J.L. Verdegay, Linear programming problems and ranking of fuzzy numbers, Fuzzy Sets and Systems 32 (1989) 1-11. 1

5. P. Fortemps and M. Roubens, Ranking and defuzzification methods based on area compensation, Fuzzy Sets and Systems 82 (1996) 319-330. 3

6. G.J. Klir and B. Yuan, Fuzzy Sets and Fuzzy Logic: Theory and Applications, PrenticeHall, PTR, New Jersey, 1995. 1

7. Y.J. Lai and C.L. Hwang, Fuzzy Mathematical Programming Methods and Applications, Springer, Berlin, 1992. 1, 2

8. N. Mahdavi-Amiri and S.H. Nasseri, Duality results and a dual simplex method for linear programming problems with trapezoidal fuzzy variables, Fuzzy Sets and Systems 158 (2007) 1961-1978. 1, 3

9. N. Mahdavi-Amiri and S.H. Nasseri, Duality in fuzzy number linear programming by use of a certain linear ranking function, Applied Mathematics and Computation 180 (2006) 206-216. 1

10. H.R. Maleki, M. Tata and M. Mashinchi, Linear programming with fuzzy variables, Fuzzy Sets and Systems 109 (2000) 21-33. 1

11. H. Tanaka, T. Okuda and K. Asai, On fuzzy mathematical programming, The Journal of Cybernetics 3 (1974) 37-46. 1

12. R.R. Yager and D.P. Filev, Essentials of Fuzzy Modeling and Control, John Wiley and Sons, New York, 1994. 3

Department of Mathematics, Mazandaran University, Babolsar, Iran.

E-mail address: nasseri@umz.ac.ir 